\title{
Effect of Left Ventricular Volume on the Magnitude of Functional Depression by External Minute Vibration
}

\author{
Yoshiro Koiwa, Nobuo Hoshi, Tadasu Ohyama, \\ Takehiko Takagi, Jun-IChi Kikuchi, Hideyuki Honda \\ and Tamotsu Takishima
}

The First Department of Internal Medicine, Tohoku University School of Medicine, Sendai 980

\begin{abstract}
Koiwa, Y., Hoshi, N., Ohyama, T., Takagi, T., Kikuchi, J., Honda, H. and Tакishima, T. Effect of Left Ventricular Volume on the Magnitude of Functional Depression by External Minute Vibration. Tohoku J. Exp. Med., 1989, 159 (2), 167-168 - We examined the effect of the left ventricular (LV) volume on the magnitude of the vibration induced functional depression (VID) using four canine cross-circulated isovolumically beating LV preparations. A sinusoidal, $50 \mathrm{~Hz}$ vibration ( $1.5 \mathrm{~mm}$ in amplitude) was applied to the ventricular anterior epicardium at different values of $\mathrm{LV}$ volume which was stepwisely increased by $1-2 \mathrm{ml}$ from 0 to $30 \mathrm{ml}$. VID was estimated as the difference of the peak LV pressure between control and during external vibration. VID increased as an increment in LV volume when the volume was below 7-8 $\mathrm{ml}$ and when peak LV pressure was less than $70 \mathrm{mmHg}$. However, VID remained constant when the volume increased further. We concluded that VID was independent of LV volume at physiological range. vibration induced depression; LV volume dependency; isolated LV preparation
\end{abstract}

The minute sinusoidal vibration of $2.17 \mathrm{~mm}$-amplitude has been reported to induce left ventricular (LV) functional depression in ejecting preparations as well as isolated LV preparations. This vibration induced functional depression (VID) has a unique feature in that it is highly sensitive to mild changes in LV inotropy of latent heart failure (Koiwa et al. 1989a). VID has been suggested to reflect the change in myocardial crossbridge kinetics and may potentially be a new measure of LV inotropy. However, it has not been examined whether the loading condition could influence the value of VID besides the basic LV inotropy. To answer this question, we examined the effect of $L V$ volume on the magnitude of VID using four cross-circulated isolated isovolumic canine LV preparations. In brief, just the upper portion of the aortic valve and mitral orifice of isolated heart from the source dog (14-16 kg, body weight) were closed to make the heart isovolumic. The right ventricular free wall and both atriae were excised, ligating with fine silk threads. The junctional pacing rate and coronary flow rate, perfused from the femoral artery of the support dog (18-24 kg, body weight) via extra corporeal line, were maintained constant throughout the experiment as $100-140$ beats $/ \mathrm{min}$ and $80-100 \mathrm{ml} / \mathrm{min} / 100 \mathrm{~g} \mathrm{LV}$, respective-

Received August 31, 1989; revision accepted for publication September 30, 1989.

Mailing address : Y. Koiwa, M.D., the First Department of Internal Medicine, Tohoku University School of Medicine, 1-1 Seiryo-machi, Aoba-ku 980, Sendai, Japan. 


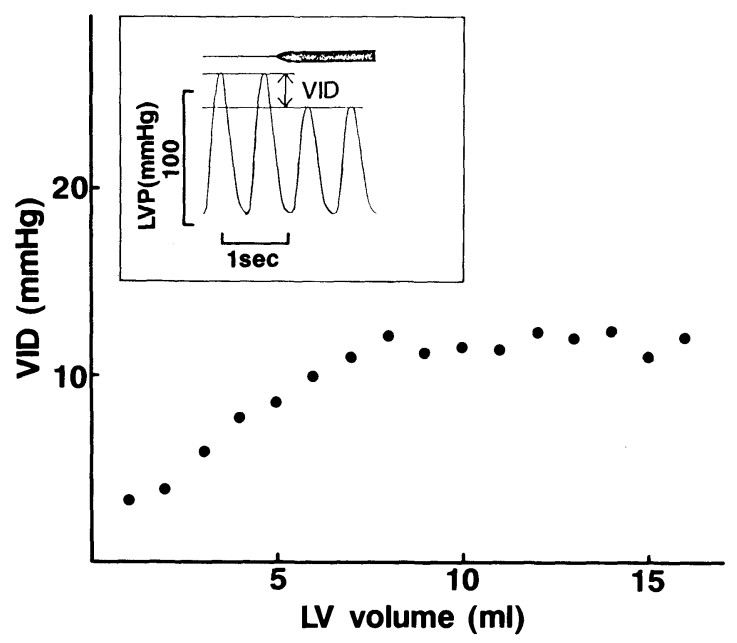

Fig. 1. The magnitude of VID at different VL volume. Two different relations between VID and LV volume were observed. That is, VID showed LV volume dependency at smaller $L V$ volume, however, at larger LV volume, VID was independent of the LV volume. Inset is actual trace of vibration signal (top) and LV pressure at control and under external vibration.

ly. LV pressure was measured with catheter tip micromanometer. ECG was also recorded with silver tip electrodes. After steady contraction was established, LV volume was stepwisely increased by 1 or $2 \mathrm{ml}$ from 0 to approx. $30 \mathrm{ml}$ by infusing warm physiological saline manually. At each value of LV volume, the epicardial surface of the isolated LV was oscillated with $50 \mathrm{~Hz}$, sinusoidal vibration ( $1.5 \mathrm{~mm}$ amplitude) by attaching the tip of the vibrator. The magnitude of VID was calculated as the difference between the peak LV pressure at control and those under external vibration. The effect of LV volume on VID differed with the range of the volume examined. That is, at smaller volume of $\simeq 8 \mathrm{ml}$ (peak LVP $<70 \mathrm{mmHg}$ ), VID increased linearly with an increment of LV volume: VID $(\mathrm{mmHg})=1.30 \times \mathrm{LV}$ volume $(\mathrm{ml})+6.11, \mathrm{r}=0.96, p<0.01 ; \mathrm{VID}=1.30 \times \mathrm{LV}$ volume +2.01 , $\mathrm{r}=0.99 . p<0.01 ; \mathrm{VID}=2.12 \times \mathrm{LV}$ volume $+9.81, \mathrm{r}=0.99, p<0.01 ; \mathrm{VID}=1.00 \times \mathrm{LV}$ volume $+0.40, \mathrm{r}=0.98, p<0.01$. However, when the LV volume increased further, the VID remained unchanged showing volume independency at this volume range (Fig. 1). Results in this study showing volume independency of VID at physiological range (see, the value of peak LV pressure would decrease to much lower value if the ventricle allowed ejection) suggested another advantage in estimating the modest change of the crossbridge kinetics by VID measurement, which seemed to underlie in the patient with latent heart failure (Koiwa et al. 1989a). This speculation for future clinical application is, of course, premature at present because other factors such as afterload or heart rate were not examined in relation to VID. However, we demonstrated that we could vibrate the volunteer's LV wall with precordially applied vibration when the respiration and body position were carefully controlled (Koiwa et al. 1989b).

\section{References}

1) Koiwa, Y., Hoshi, N., Ohyama, T., Takagi, T., Kikuchi, J., Honda, H. \& Takishima, T. (1989a) Evaluation of left ventricular function by application of external minute vibration. Front. Med. Biol. Engng., in press.

2) Koiwa, Y., Kikuchi, J., Takagi, T., Honda, H., Hoshi, N. \& Takishima, T. (1989b) Human left ventricular wall vibration responded to precordial minute vibration. Tohoku J. Exp. Med., 1989, 159, 79-80. 